\title{
Radiosensitivity of human ovarian carcinoma and melanoma cells to $\gamma$-rays and protons
}

\author{
Otilija Keta ${ }^{1}$, Danijela Todorović ${ }^{2}$, Nataša Popović ${ }^{1}$, Lela Korićanac ${ }^{1}$, Giacomo Cuttone ${ }^{3}$, \\ Ivan Petrović ${ }^{1}$, Aleksandra Ristić-Fira ${ }^{1}$
}

${ }^{1}$ Vinča Institute of Nuclear Sciences, University of Belgrade, Belgrade, Serbia
${ }^{2}$ Medical Faculty, University of Kragujevac, Kragujevac, Serbia
${ }^{3}$ Istituto Nazionale di Fisica Nucleare, Laboratori Nazionali del Sud, Catania, Italy

Submitted: 11 June 2012

Accepted: 24 February 2013

Arch Med Sci 2014; 10, 3: 578-586

DOI: 10.5114 /aoms.2014.43751

Copyright @ 2014 Termedia \& Banach

\section{Abstract}

Introduction: Proton radiation offers physical advantages over conventional radiation. Radiosensitivity of human 59M ovarian cancer and HTB140 melanoma cells was investigated after exposure to $\gamma$-rays and protons.

Material and methods: Irradiations were performed in the middle of a $62 \mathrm{MeV}$ therapeutic proton spread out Bragg peak with doses ranging from 2 to $16 \mathrm{~Gy}$. The mean energy of protons was $34.88 \pm 2.15 \mathrm{MeV}$, corresponding to the linear energy transfer of $4.7 \pm 0.2 \mathrm{keV} / \mu \mathrm{m}$. Irradiations with $\gamma$-rays were performed using the same doses. Viability, proliferation and survival were assessed 7 days after both types of irradiation while analyses of cell cycle and apoptosis were performed $48 \mathrm{~h}$ after irradiation.

Results: Results showed that $\gamma$-rays and protons reduced the number of viable cells for both cell lines, with stronger inactivation achieved after irradiation with protons. Surviving fractions for $59 \mathrm{M}$ were $0.91 \pm 0.01$ for $\gamma$-rays and $0.81 \pm 0.01$ for protons, while those for HTB140 cells were $0.93 \pm 0.01$ for $\gamma$-rays and $0.86 \pm 0.01$ for protons. Relative biological effectiveness of protons, being $2.47 \pm 0.22$ for $59 \mathrm{M}$ and $2.08 \pm 0.36$ for HTB140, indicated that protons provoked better cell elimination than $\gamma$-rays. After proton irradiation proliferation capacity of the two cell lines was slightly higher as compared to $\gamma$-rays. Proliferation was higher for 59M than for HTB140 cells after both types of irradiation. Induction of apoptosis and G2 arrest detected after proton irradiation were more prominent in $59 \mathrm{M}$ cells.

Conclusions: The obtained results suggest that protons exert better antitumour effects on ovarian carcinoma and melanoma cells than $\gamma$-rays. The dissimilar response of these cells to radiation is related to their different features.

Key words: apoptosis, cell cycle, $\gamma$-rays, melanoma, protons, ovarian carcinoma.

\section{Introduction}

Ionizing radiation initiates diverse biochemical processes that determine the cellular response. DNA repair mechanisms, cell cycle arrest and apoptosis are probably the most important biological events [1]. Radiation therapy is among first-line treatments in curing cancer [2]. However, it is well known that various tumours respond differently to the same type of treatment. For example, lymphomas and leukaemias are generally highly radiosensitive, squamous cell carcinomas show intermediate

\author{
Corresponding author: \\ Aleksandra Ristić-Fira \\ Vinča Institute \\ of Nuclear Sciences \\ University of Belgrade \\ P.O. Box 522 \\ 11001 Belgrade, Serbia \\ Phone/fax: +38111645 5561 \\ E-mail: aristic@vin.bg.ac.rs
}


sensitivity, while melanomas are considered to be the most resistant to radiation therapy, as well as to other therapeutic agents [3-5]. The same type of treatment can induce different responses even among tumours of the same histological type [6]. This differential sensitivity of the tumour cells and their ability to recover from radiation damage explains why conventional radiation therapy can be successfully used to control certain types of tumours, while having less success in others [7].

DNA is widely recognized to be the most important target for ionizing radiation. Thus, exposure to radiation results directly in DNA injuries or indirectly by ionizing cellular water molecules to produce free radicals that are involved in the DNA damage [1]. Various types of cytotoxic DNA lesions induced by ionizing radiation are classified into DNA double-strand breaks (DSBs), DNA single-strand breaks (SSBs), DNA adducts and DNA-protein cross-links [8]. The DNA DSBs are generally considered to be critical DNA lesions with greater biological significance than SSBs. They have important biological consequences such as cell death, chromosome aberration and loss of genetic information [9].

The aim of radiation therapy is to kill tumour cells and to avoid damage of the neighbouring normal tissue. Proton radiation offers several advantages over conventional photon radiation including the ability to deliver much higher specific energy loss per unit length, i.e. linear energy transfer (LET), precisely to the tumour volume, while sparing the surrounding healthy tissue [10]. Due to the physical properties of protons maximum ionization is achieved at the precisely controlled position, just before the end of the range, which is known as the Bragg peak. In order to deliver a uniform dose to the entire tumour volume the Bragg peak is spread out by modifying proton energy at the price of a moderately increased entrance dose [11]. The relative biological effectiveness (RBE) reflects biological effectiveness of the specific type of ionizing radiation in comparison to the reference radiation.

In this study, the level of cellular response of irradiated human 59M ovarian cancer and human HTB140 melanoma cells was investigated and compared using assays for the assessment of cell viability, proliferation, cell cycle and apoptosis. Inactivation effects of the two different radiation qualities, $\gamma$-rays and protons, were analysed for each cell line.

\section{Material and methods}

\section{Cell culture}

The human 59M and HTB140 cells were purchased from the American Tissue Culture Collec- tion (Rockville, MD, USA) and routinely maintained as a monolayer in RPMI1640 medium supplemented with $10 \%$ fetal bovine serum, penicillin/ streptomycin and L-glutamine (all from Sigma-Aldrich Chemie $\mathrm{GmbH}$, Steinheim, Germany) under standard conditions at $37^{\circ} \mathrm{C}$ in humidified atmosphere with $5 \% \mathrm{CO}_{2}$ (Heraeus, Hanau, Germany).

\section{Irradiation conditions}

Proton irradiations were performed within the spread-out Bragg peak (SOBP) of the $62 \mathrm{MeV}$ therapeutic proton beam produced by the superconducting cyclotron at the CATANA (Centro di Adro Terapia e Applicazioni Nucleari Avanzate) treatment facility in the Istituto Nazionale di Fisica Nucleare (INFN), Laboratori Nazionali del Sud (LNS) in Catania, Italy. Irradiation position was in the middle of the SOBP. This was achieved by interposing a $16.3 \mathrm{~mm}$ thick Perspex plate (polymethyl methacrylate-PMMA) between the final collimator and cell monolayer, thus obtaining a corresponding relative dose of $99.42 \pm 0.58 \%$. The mean energy of protons was $34.88 \pm 2.15 \mathrm{MeV}$, corresponding to the LET of $4.7 \pm 0.2 \mathrm{keV} / \mu \mathrm{m}$. Reference dosimetry was performed using a plane-parallel PTW 34045 Markus ionization chamber calibrated according to International Atomic Energy Agency technical Report series (IAEA 2000) code of practice $[12,13]$. Single doses delivered to the cells were 2, 4, 8, 12 and $16 \mathrm{~Gy}$, at the dose rate of $15 \mathrm{~Gy} / \mathrm{min}$.

Irradiations with ${ }^{60} \mathrm{Co} \gamma$-rays at the same dose level and with the dose rate of $1 \mathrm{~Gy} / \mathrm{min}$ were performed at the Vinča Institute of Nuclear Sciences, Belgrade, Serbia. All irradiations were carried out in air at room temperature. Cell viability and cell proliferation assays were performed 7 days after irradiation under standard conditions. The results were presented as the percentage of control values. Cell cycle distribution and induction of apoptosis were evaluated $48 \mathrm{~h}$ after irradiation.

\section{Cell viability assays}

Cell viability was estimated 7 days after irradiation using Cell Proliferation Kit I, MTT (Roche Diagnostics GmbH, Mannheim, Germany) according to the manufacturer's procedure. The absorbance was measured using a microplate reader (Wallac, VICTOR2 1420 Multilabel counter, PerkinElmer, Turku, Finland) at a test wavelength of $550 \mathrm{~nm}$ and a reference wavelength of $690 \mathrm{~nm}$.

The sulforhodamine B (SRB) assay, based on the measurement of cellular protein content, was used for cell density determination. Cells were seeded in 96-well plates (Nunclon ${ }^{\mathrm{TM}}$, Roskilde, Denmark), at the appropriate concentration. After 7 days of post-irradiation incubation, cell monolayers were fixed with $10 \%$ trichloroacetic acid (TCA) for $1 \mathrm{~h}$ 
at $4^{\circ} \mathrm{C}$. Plates were then washed with water and stained with $0.4 \%$ SRB (Sigma-Aldrich Chemie $\mathrm{GmbH}$ ) in $1 \%$ acetic acid for $15 \mathrm{~min}$ at room temperature. Excess stain was removed by washing repeatedly with $1 \%$ acetic acid. Plates were than air dried and the protein-bound dye was dissolved in $10 \mathrm{mM}$ Tris base solution for $5 \mathrm{~min}$. Absorbance was measured at $550 \mathrm{~nm}$ and a reference wavelength of $690 \mathrm{~nm}$ using a microplate reader (Wallac, VICTOR2 1420 Multilabel counter).

\section{Cell proliferation assays}

The DNA synthesis and cell proliferation were estimated using a 5-bromo-2-deoxyuridine (BrdU) assay (Roche Diagnostics $\mathrm{GmbH}$ ). Cells were irradiated and incubated for 7 days before determination of cell proliferation according to the manufacturer's instructions. The absorbance was measured using a microplate reader at a test wavelength of $450 \mathrm{~nm}$ and a reference wavelength of $690 \mathrm{~nm}$ (Wallac, VICTOR2 1420 Multilabel counter).

\section{Survival assay}

For the evaluation of survival after irradiation the cells were harvested by trypsinization, seeded into $25 \mathrm{~cm}^{2}$ flasks at a suitable number and incubated at $37^{\circ} \mathrm{C}$ for 7 days. Subsequently, the cells were fixed with methanol and stained with $10 \%$ Giemsa solution. Groups of at least fifty cells were scored as individual colonies.

\section{Flow cytometric analysis of cell cycle}

For the flow cytometric evaluation of the cell cycle status $1 \times 10^{6}$ cells were taken from each flask, washed with phosphate buffered saline (PBS), fixed overnight with $70 \%$ cold ethanol and stained with PBS buffer that contained $50 \mu \mathrm{g} / \mathrm{ml}$ propidium iodide (PI) and $50 \mu \mathrm{g} / \mathrm{ml}$ RNase (both from Sigma-Aldrich Chemie $\mathrm{GmbH}$ ). After incubation at room temperature for $30 \mathrm{~min}$, cells were analysed by flow cytometry (FACSCalibur; Becton Dickinson, USA) using ModFit software.

\section{Flow cytometric analysis of apoptosis}

For quantification of the apoptotic cells, flow cytometric analysis with the Annexin V-FLUOS Staining kit (Roche Diagnostics $\mathrm{GmbH}$ ) was performed according to the manufacturer's instructions. Cells were trypsinized, washed in ice-cold PBS and resuspended in $100 \mu \mathrm{l}$ Annexin V-Fluos labelling solution (containing Annexin V-Fluos labelling reagent and $\mathrm{PI}$ ). After their incubation in the dark for $30 \mathrm{~min}, 500 \mu \mathrm{l}$ of an incubation buffer was added per sample. Samples were analysed on a FACSCalibur cytometer (Becton Dickinson). For each sample, 10000 cells were analysed. The number of apoptotic cells was calculated using the ModFit software (Verity Software, Becton Dickinson).

\section{Statistical analysis}

Quadruplicate measurements were made during each experiment, and all experiments were repeated at least twice. Independent Student's $t$-test was performed to assess the significance of differences among analysed experimental groups. The level of significance was set at $p<0.05$. Results were presented as the mean \pm SD (standard deviation).

\section{Results}

\section{Effects of $\gamma$-rays and protons on cell viability}

Exponentially growing 59M and HTB140 cells were irradiated with single doses (2 to $16 \mathrm{~Gy}$ ) of $\gamma$-rays and protons. To compare inactivation ability of relatively high LET proton irradiation with $\gamma$-rays, the level of cell viability was measured 7 days after irradiation by MTT and SRB tests, as well as by clonogenic assay.

The viability data for the $59 \mathrm{M}$ cells obtained by MTT assay revealed good dose-dependent inhibitory effects for both types of radiation. However, better inactivation was detected after proton irradiation ( $p<0.05$ for $16 \mathrm{~Gy}$ ). According to the MTT assay cell viability ranged from $88 \%$ to $47 \%$ for $\gamma$-rays ( $p<0.05$ for 2 Gy, $p<0.001$ for higher doses) and from $82 \%$ to $32 \%$ for protons ( $p<0.01$ for $2 \mathrm{~Gy}, p<0.001$ for higher doses), as shown in Figure $1 \mathrm{~A}$. Cell viability evaluated by SRB assay and presented in Figure $1 \mathrm{~B}$ was in the interval from $94 \%$ to $71 \%$ for $\gamma$-rays ( $p<0.05$ for $4 \mathrm{~Gy}$, $p<0.001$ for higher doses) and from $86 \%$ to $51 \%$ for proton irradiation ( $p<0.01$ for $2 \mathrm{~Gy}, p<0.001$ for higher doses). The SRB assay also showed better dose-dependent inhibitory effects after proton irradiation as compared to $\gamma$-rays ( $p<0.05$ for 12 and $16 \mathrm{~Gy})$. These results indicated that the SRB assay provided somewhat higher values as compared to MTT.

Very good agreement of dose-dependent viability data was obtained between MTT and SRB assays for the HTB140 cells. Estimated cell viability assessed by MTT was from $87 \%$ to $63 \%$ after $\gamma$-rays ( $p<0.05$ for 2 Gy, $p<0.001$ for higher doses), while for protons it was from $69 \%$ to $34 \%$ $(p<0.001)$, and is shown in Figure $2 \mathrm{~A}$. The values obtained by SRB assay, which are given in Figure $2 \mathrm{~B}$, ranged from $85 \%$ to $74 \%$ after $\gamma$-rays ( $p<0.01$ for $2 \mathrm{~Gy}, p<0.001$ for higher doses) and from $72 \%$ to $40 \%$ after proton irradiation $(p<0.001)$. A stronger dose-dependent radiation response was obtained by both assays after proton irradiation than after $\gamma$-rays $(p<0.001)$.

Results obtained for the two cell lines by clonogenic assay that was performed 7 days after irradia- 
A

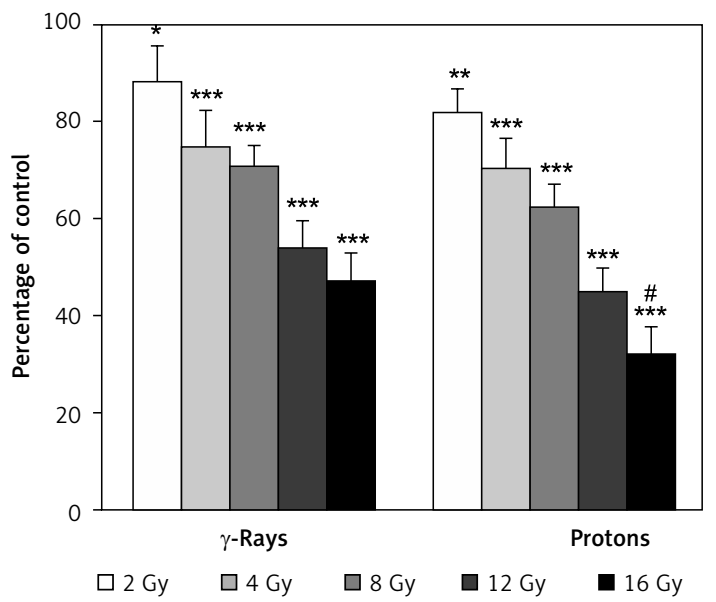

B

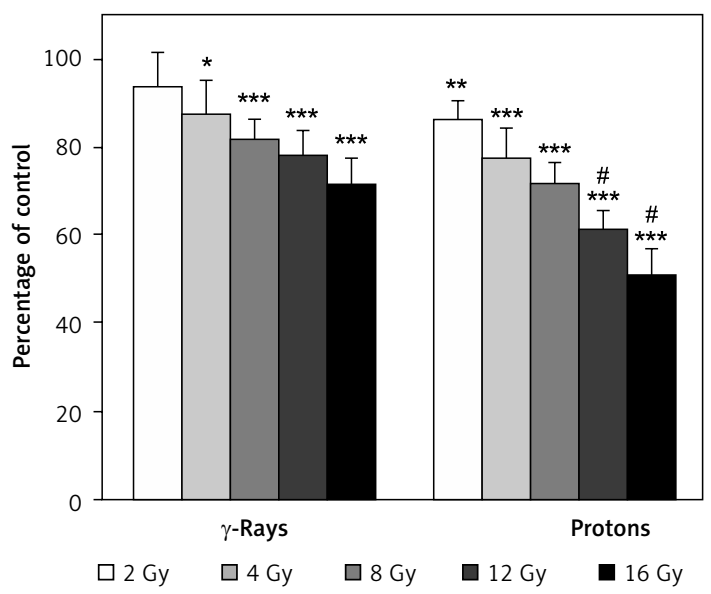

Figure 1. Effects of $\gamma$-rays and protons on $59 \mathrm{M}$ cell viability 7 days after irradiation, estimated by MTT (A) and SRB assays (B). Data obtained from 4 experiments are presented as means \pm SD. Irradiation doses were $2-16 \mathrm{~Gy}$ ${ }^{*}$ Statistical significance compared to the control, "statistical significance compared to $\gamma$-rays; " * $0.01<p<0.05,{ }^{*, * \#} 0.001<p<$ $0.01,{ }^{* * *}, \# \# p<0.001$

A

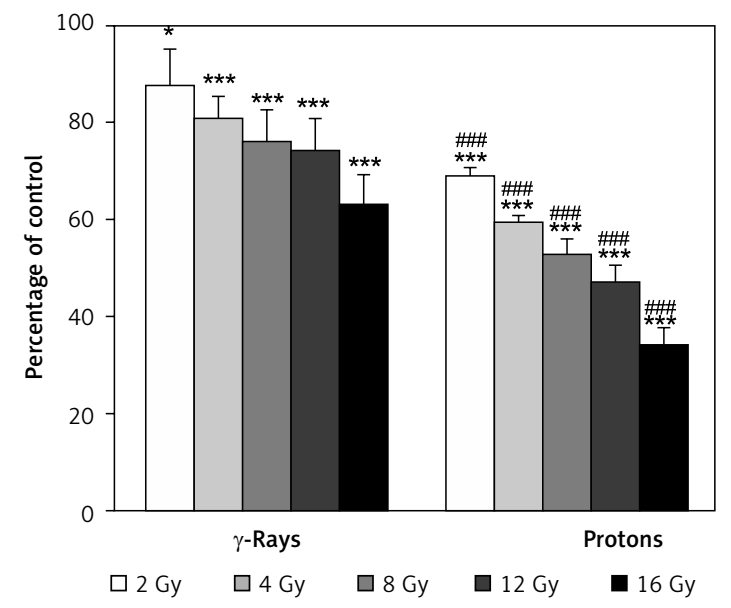

B

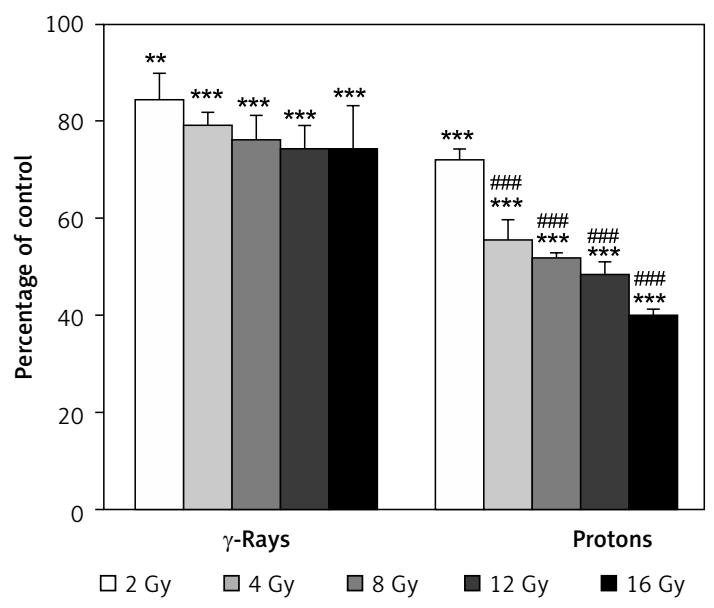

Figure 2. Effects of $\gamma$-rays and protons on HTB140 cell viability 7 days after irradiation, estimated by MTT (A) and SRB assays (B). Data obtained from 4 experiments are presented as means \pm SD. Irradiation doses were 2-16 Gy ${ }^{*}$ Statistical significance compared to the control, " statistical significance compared to $\gamma$-rays, , $, 0.01<p<0.05,{ }^{* *}, \# 0.001<p<0.01$, ***\#\#, $p<0.001$

tions with protons and $\gamma$-rays are presented in Figure 3. Survival data were fitted using the linear-quadratic equation: $S=\exp \left(-\alpha D-\beta D^{2}\right)$, where $S$ is the surviving fraction for the dose $D$, while $\alpha$ and $\beta$ are fitting parameters. The values of fitting parameter $\alpha$, coefficient of determination $\left(r^{2}\right)$ and fit standard error are given in Table I. Due to very low values, parameter $\beta$ is not included in Table I.

The fitted curves revealed that the level of cell inactivation produced by proton irradiation was higher than that of $\gamma$-rays for both cell lines. The killing capacity of both radiation types was more pronounced for the $59 \mathrm{M}$ cells (Figure $3 \mathrm{~A}$ ) than for the HTB140 cells (Figure 3 B). Surviving fractions at 2 Gy, i.e. SF2 values, reflecting the level of cellular radiosensitivity, were obtained from the fitted curves and are given in Table I. According to these results the SF2 values for the ovarian $59 \mathrm{M}$ cells were 0.91 \pm 0.01 and $0.81 \pm 0.01$ for $\gamma$-rays and protons, respectively. The HTB140 cells expressed somewhat higher level of radioresistance, with the SF2 values of 0.93 \pm 0.01 for $\gamma$-rays and $0.86 \pm 0.01$ for protons.

The RBE values, defined as the ratio of 2 Gy $\gamma$-rays dose and the proton dose producing the same inactivation level as that given by 2 Gy of the reference $\gamma$-rays, were $2.47 \pm 0.22$ for the $59 \mathrm{M}$ and $2.08 \pm 0.36$ for the HTB140 cells (Table I).

\section{Effects of $\gamma$-rays and protons on cell proliferation}

The effects of $\gamma$-rays and protons on cell proliferation of 59M and HTB140 cells are presented in 
A

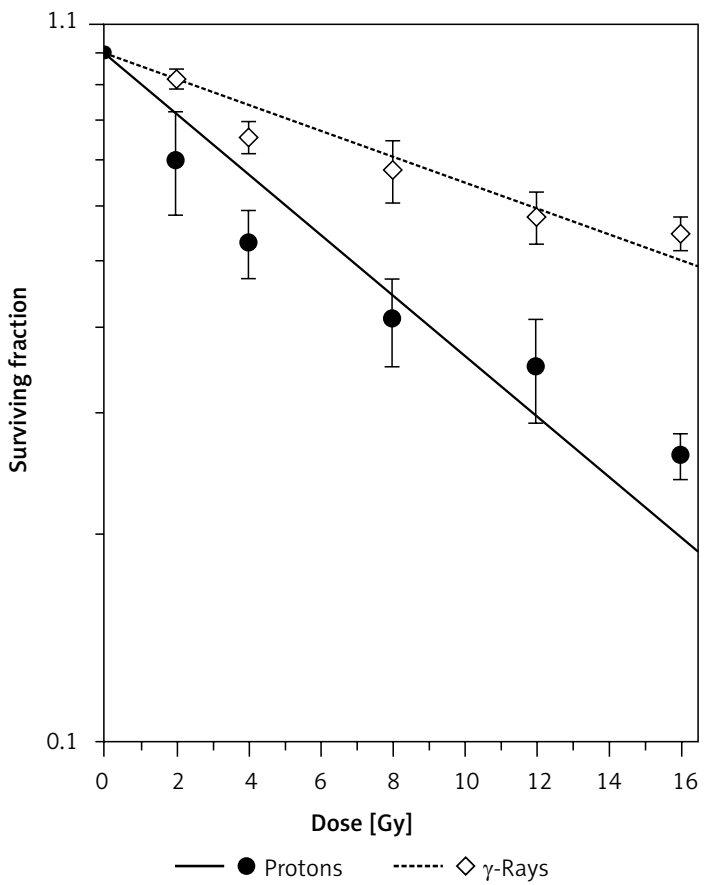

B

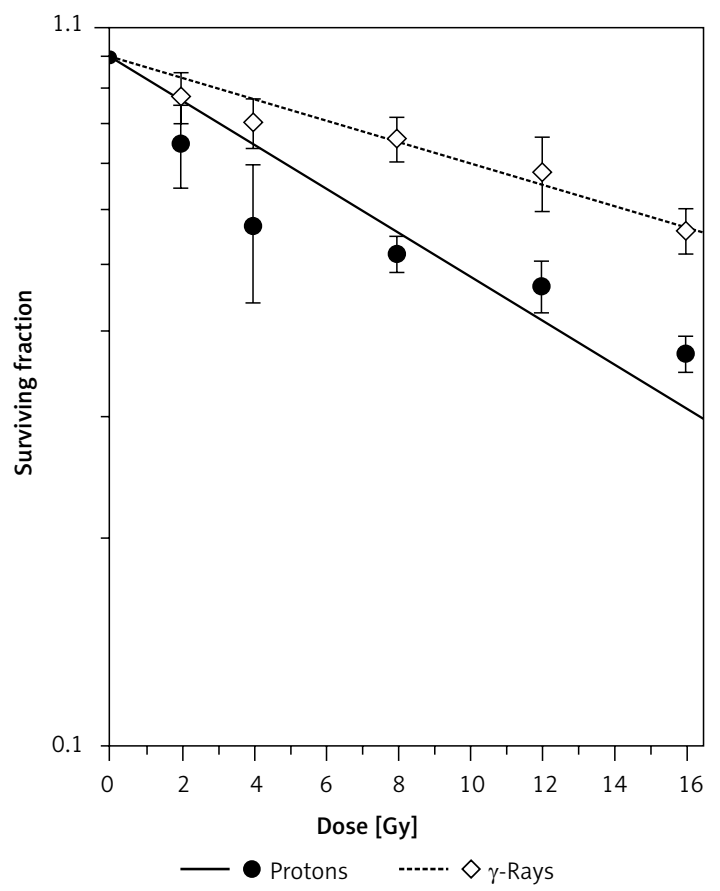

Figure 3. Dose-response curves of 59M cells (A) and HTB140 cells (B) exposed to $\gamma$-rays and protons, obtained by clonogenic assay. Irradiation doses were 2-16 Gy. Data obtained from 4 experiments are presented as means \pm SD

Table I. Fitting parameters, SF2 and RBE (2 Gy, $\gamma$ ) for 59M and HTB140 cells after irradiation with $\gamma$-rays and protons

\begin{tabular}{|c|c|c|c|c|c|}
\hline Irrad. type & $\alpha\left(G^{-1}\right)$ & $r^{2}$ & Fit Std. Err. & SF2 & RBE $(2 \mathrm{~Gy}, \gamma)$ \\
\hline \multicolumn{6}{|l|}{$59 M$} \\
\hline$\gamma$-Rays & $0.043 \pm 0.012$ & 0.92 & 0.06 & $0.91 \pm 0.01$ & 1.00 \\
\hline Protons & $0.101 \pm 0.015$ & 0.89 & 0.10 & $0.81 \pm 0.01$ & $2.47 \pm 0.22$ \\
\hline \multicolumn{6}{|l|}{ HTB 140} \\
\hline$\gamma$-Rays & $0.036 \pm 0.009$ & 0.93 & 0.05 & $0.93 \pm 0.01$ & 1.00 \\
\hline Protons & $0.073 \pm 0.012$ & 0.80 & 0.12 & $0.86 \pm 0.01$ & $2.08 \pm 0.36$ \\
\hline
\end{tabular}

$r^{2}$-coefficient of determination $=1-$ sum of squares about the mean/sum of squared errors. Results are presented as the mean \pm standard deviation

Figure 4. There was a trend of a dose-dependent decrease of proliferation for each cell line after exposure to the two radiation types.

Proliferation capacity of $59 \mathrm{M}$ cells was in the range from $80 \%$ to $45 \%$ for $\gamma$-rays $(p<0.001)$ and from $89 \%$ to $53 \%$ for protons ( $p<0.05$ for $2 \mathrm{~Gy}$, $p<0.001$ for higher doses). A very pronounced dose-dependent decrease of proliferation was observed, having rather high values in the whole dose range (Figure $4 \mathrm{~A}$ ). The level of cell proliferation of HTB140 cells was from $46 \%$ to $35 \%$ for $\gamma$-rays $(p<0.001)$ and from $50 \%$ to $44 \%$ for protons ( $p<$ $0.001)$. The decrease of proliferation with dose was minor with rather low values for all doses (Figure 4 B).

\section{Effects of $\gamma$-rays and protons on cell cycle and apoptosis}

In order to obtain an in-depth picture of antitumour effects of $\gamma$-rays and protons, cell cycle and apoptosis were investigated. Since the dose of $8 \mathrm{~Gy}$ of proton radiation inhibited survival of analysed cell lines close to $50 \%$, this dose was chosen for the analyses. Literature data suggested that for the majority of cells apoptosis occurs after one or more cell divisions. Usually the maximum number of apoptotic cells in in vitro conditions can be found $48 \mathrm{~h}$ after irradiation [14]. Based on this fact, the time point of $48 \mathrm{~h}$ after treatment was selected for the assessment of apoptosis in this study. Accordingly, changes in cell cycle distribution were determined at the same time point.

Both types of radiation induced an increase of G2 cell population in $59 \mathrm{M}$ cells as compared to untreated control ( $p<0.05$ for protons). This was followed by a reduction of $\mathrm{S}$ phase. More prominent changes were observed after irradiation with protons (Figure $5 \mathrm{~A}$ ). Similar trends were found in irradiated HTB140 melanoma cells (Figure 5 B). 
A

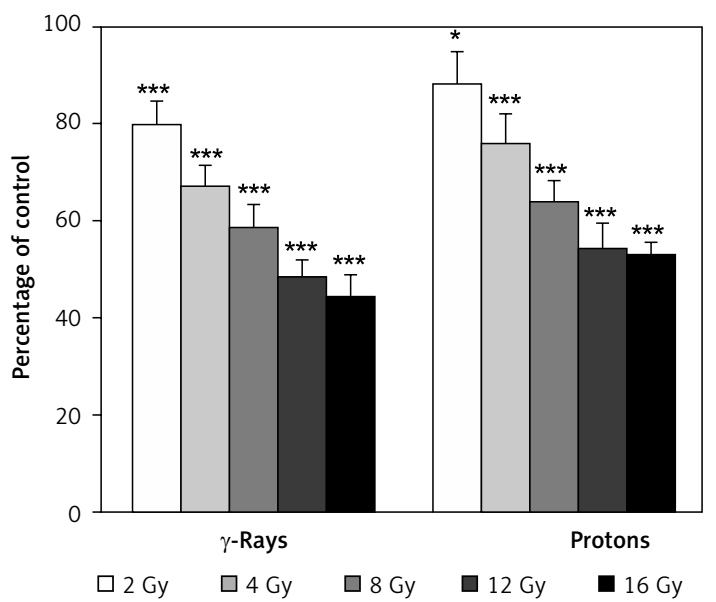

B

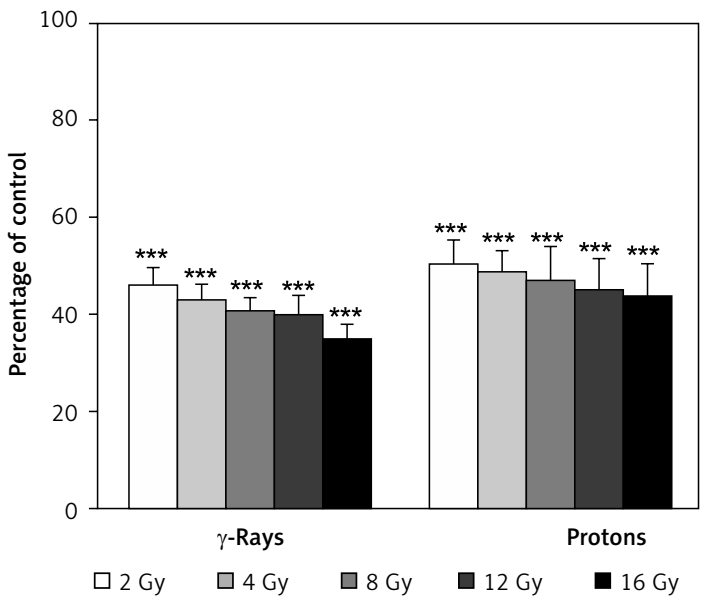

Figure 4. Dose-dependent cell proliferation of 59M cells (A) and HTB140 cells (B) estimated by BrdU assay. Data obtained from 4 experiments are presented as means \pm SD. Irradiation doses were $2-16$ Gy

*Statistical significance compared to the control, " ${ }^{*}$ statistical significance compared to $\gamma$-rays, , , $0.01<p<0.05,{ }^{* *}, \#$ \# $0.001<p<$

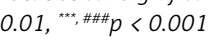

A

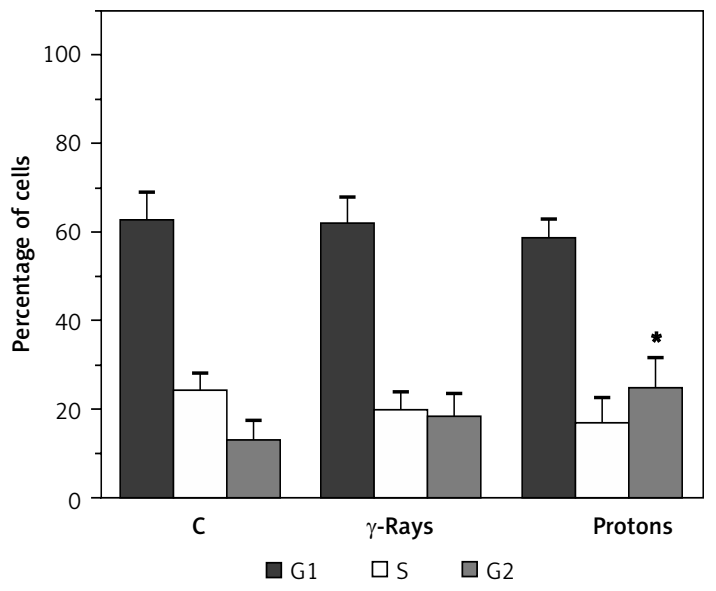

B

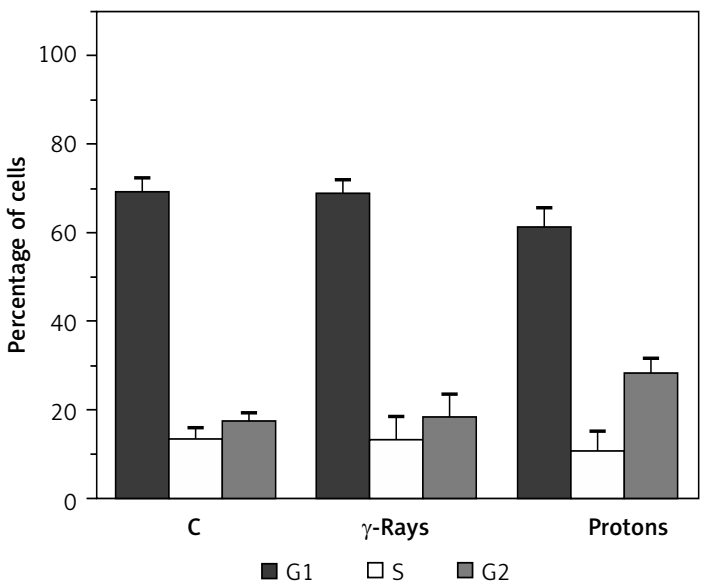

Figure 5. The effects of $\gamma$-rays and proton radiation on cell cycle distribution in 59M (A) and HTB140 (B) cell line, estimated by flow cytometry $48 \mathrm{~h}$ after irradiation. Irradiation dose was $8 \mathrm{~Gy}$. Data obtained from 4 experiments are presented as means \pm SD

*Statistical significance compared to the control, " statistical significance compared to $\gamma$-rays, ${ }^{*}, 0.01<p<0.05,{ }^{* *}, \# 0.001<p<0.01$, $* * \#, \#<0.001$

The obtained results indicated that $\gamma$-rays did not induce a significant increase of apoptosis in either 59M or HTB140 cells. However, proton radiation showed a better pro-apoptotic effect than $\gamma$-rays $(p<0.001$ for 59M, $p<0.01$ for HTB140 cells). The percentage of induced apoptosis after irradiation with protons was $22 \%$ in $59 \mathrm{M}$ cells ( $p<0.001$, as compared to control), and $18 \%$ in HTB140 cells ( $p<0.001$, as compared to control) (Figure 6).

\section{Discussion}

Many assays have been developed for the prediction of individual radiosensitivity of tumour cells, but they have practical limitations. Despite disadvantages of the clonogenic assay such as its long duration and limitations with respect to the ability of cells to form colonies, the clonogenic assay is still considered to be the "gold standard" for the evaluation of cellular radiosensitivity [15]. The colorimetric microtetrazolium (MTT) assay is based on the ability of viable cells to reduce 3-(4,5-dimethylthiazol-2-yl)-2,5-diphenyltetrazolium bromide, a soluble yellow tetrazolium salt into blue-purple formazan crystals, thus overcoming the need for cells to form colonies. However, this behaviour is not linear at high cell densities [16]. Moreover, the SRB protein binding assay based on quantifying total protein content of viable cells 


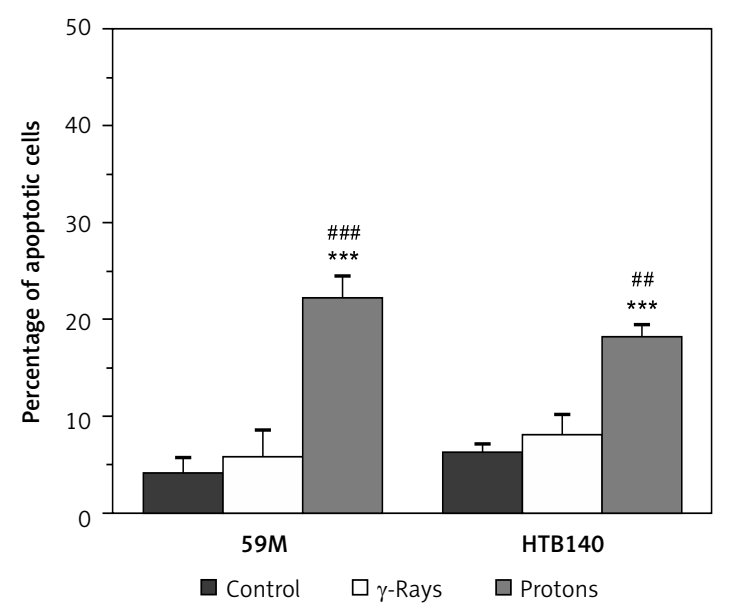

Figure 6. The effects of $\gamma$-rays and proton radiation on apoptosis in 59M and HTB140 cell line, estimated by flow cytometry $48 \mathrm{~h}$ after irradiation. Irradiation dose was 8 Gy. Data obtained from 4 experiments are presented as means \pm SD

*Statistical significance compared to the control, "statistical significance compared to $\gamma$-rays, ${ }^{*}, 0.01<p<0.05,{ }^{* *}, \# 0.001$

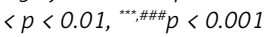

has some advantages over the MTT assay, such as higher sensitivity and linearity even at greater cell densities [17]. Unlike the MTT, the results obtained with the SRB assay are independent of metabolic conditions of the cells [18]. In addition, the BrdU assay, designed to follow DNA replication, is frequently used to assess proliferation capacity of irradiated cells [19].

Comparing the two cell lines, MTT and SRB assays detected a rather similar response after proton irradiation, while the dose-dependent response after $\gamma$-rays appears to be more pronounced for the $59 \mathrm{M}$ cells. Although having the same dose-dependent trend in the cell inactivation, certain differences in the results obtained by MTT and SRB were observed. Different criteria that are used in determination of cell viability by these two assays could be responsible for these differences. The MTT assay detects just living cells because it records only the cells with preserved mitochondrial function. In contrast, the SRB assay measures the total protein content and is independent of metabolic conditions of the cell line. This assay does not need specific optimization for each individual cell line [20]. Therefore, slightly lower cell inactivation that was detected by SRB could be explained by possible presence of the proteins coming from the dead cells.

According to the SF2 values acquired from the fitted curves, the HTB140 cells have a higher level of radioresistance to $\gamma$-rays and protons than the $59 \mathrm{M}$ cell line. Still, both cell lines are among very radioresistant cells. Within each cell line protons were more efficient in cell inactivation than $\gamma$-rays, as illustrated by the corresponding RBE values.
Moreover, the RBE of the $59 \mathrm{M}$ cells was greater than that of the HTB140 cells, which is due to stronger elimination of the $59 \mathrm{M}$ cells by protons. Very high radioresistance of HTB140 cells was discussed in a previous radiosensitivity study [4, 11, 21]. The SF2 values obtained for the HTB140 cells indicated that these cells are very high on the radiosensitivity scale reported for other cell lines [22]. A similar level of radioresistance to $\gamma$-rays was reported for three human ovarian cell lines [23]. Moreover, the level of radioresistance of the HTB140 cells was almost the same when compared to data reported for the U1 human bladder carcinoma cell line [24].

Analysing simultaneously the results of cell survival and proliferation within each cell line, it is apparent that they have a decreasing trend with the rise of the applied doses. Although it may seem contradictory that in the case of survival protons produce stronger cell elimination than $\gamma$-rays, while cell proliferation is somewhat higher for protons than for $\gamma$-rays, such behaviour is consistent with the physical properties of $\gamma$-rays and protons, i.e., LET and track structure [25]. Proton irradiation, due to large LET, is considered as high ionizing radiation and performs better cell elimination with respect to $\gamma$-rays. As shown in Figure 3, protons have stronger killing capacity, producing more irreparable and less reparable lesions than $\gamma$-rays. Due to their sparse truck structure $\gamma$-rays produce more reparable lesions leading to slightly lower cell proliferation as compared to protons (Figure 4). Rather different distribution of cell proliferation with the rise of the doses, for the two cell lines, is based on their dissimilar nature and some distinction in their radiosensitivity [11].

Growth inhibition of irradiated ovarian carcinoma and melanoma cells, particularly observed after irradiation with protons, can be ascribed at least partially to changes in cell cycle distribution and apoptosis. Namely, in both analysed cell lines, G2 arrest typical for irradiated cells was observed, with a significant increase noticed in $59 \mathrm{M}$ cells. This arrest is the outcome of radiation-induced DNA double strand breaks (DSBs). In our previous study [26] induction of DSBs in HTB140 melanoma cells irradiated with $\gamma$-rays was analysed through evaluation of phosphorylation of histone $\mathrm{H} 2 \mathrm{AX}$ in a time-dependent manner. Within this study expression of p21 protein was also investigated. Phosphorylation of H2AX increased after irradiation, reaching the highest value at $2 \mathrm{~h}$ after irradiation. Prolongation of post-irradiation incubation caused a reduction of phosphorylated $\mathrm{H} 2 \mathrm{AX}$ to the pre-irradiation level with a simultaneous increase of p21 protein. Since p21 is involved in the regulation of $\mathrm{G} 1$, as well as $\mathrm{G} 2$ arrest [27], these data imply that such behaviour is one of the 
causes of $\mathrm{G} 2$ arrest. Given that protons have higher LET than $\gamma$-rays, with an RBE value $\sim 2$, they induce higher numbers of DSBs in analysed cell lines and consequently more pronounced $\mathrm{G} 2$ block, as observed in our results presented herein.

An increased number of apoptotic cells was detected in both cell lines after proton irradiation. A more detailed radiation-induced mechanism of apoptosis, including changes in p53, Bax, Bcl-2 and PARP protein, as well as activation of caspase-3, was previously reported $[28,29]$. Observed induction of apoptosis was in the range characteristic for other melanoma and ovarian carcinoma cells $[30,31]$ and confirms the high level of radioresistance of analysed cell lines.

In conclusion, the two human cancer cell lines 59M ovarian and HTB140 melanoma cells revealed high levels of cellular radioresistance to $\gamma$-rays and protons. Still, HTB140 melanoma cells displayed a higher level of radioresistance, thus being among extremely resistant cell lines. Obtained radiobiological results indicated that protons, due to their high LET, are superior in cell inactivation compared to $\gamma$-rays. Due to the different nature of the cells, after irradiation with $\gamma$-rays and protons, the $59 \mathrm{M}$ cells showed stronger proliferation, as well as a higher level of apoptosis and more prominent $\mathrm{G} 2$ arrest than the HTB140 cells.

\section{Acknowledgments}

This work was supported by the Ministry of Education, Science and Technological Development of Serbia (grants 173046, 171019) and Istituto Nazionale di Fisica Nucleare, Laboratori Nazionali del Sud, Italy.

\section{References}

1. Gordon AT, McMillan TJ. A role for molecular radiobiology in radiotherapy? Clin Oncol (R Coll Radiol) 1997; 9: 70-8.

2. Steel GG. Introduction: The significance of radiobiology for radiotherapy. In: Steel GG (eds.). Basic clinical radiobiology. Edward Arnold, London1993; 1-7.

3. Joks M, Myśliwiec K, Lewandowski K. Primary breast lymphoma - a review of the literature and report of three cases. Arch Med Sci 2011; 7: 27-33.

4. Petrović I, Ristić-Fira A, Todorović D, Valastro L, Cirrone P, Cuttone G. Radiobiological analysis of human melanoma cells on the $62 \mathrm{MeV}$ CATANA proton beam. Int J Radiat Biol 2006; 82: 251-65.

5. Soengas MS, Lowe SW. Apoptosis and melanoma chemoresistance. Oncogene 2003; 22: 3138-51.

6. El-Awady RA, Dikomey E, Dahm-Daphi J. Radiosensitivity of human tumour cells is correlated with the induction but not with the repair of DNA double-strand breaks. $\mathrm{Br}$ J Cancer 2003; 89: 593-601.

7. Weichselbaum RR, Epstein J, Little JB, Kornblith P. Inherent cellular radiosensitivity of human tumors of varying clinical curability. Am J Roentgenol 1976; 127: 1027-32.
8. Forma E, Brys M, Krajewska WM. TopBP1 in DNA damage response. In: DNA repair. Dr. Inna Kruman (ed.). InTech 2011; 281-304.

9. Shikazono N, Noguchi M, Fujii K, Urushibara A, Yokoya A. The yield, processing, and biological consequences of clustered DNA damage induced by ionizing radiation. J Radiat Res (Tokyo) 2009; 50: 27-36.

10. Ristic-Fira AM, Todorovic DV, Koricanac LB, et al. Response of a human melanoma cell line to low and high ionizing radiation. Ann N Y Acad Sci 2007; 1095: 165-74.

11. Petrović I, Ristić-Fira A, Todorović D, et al. Response of a radioresistant human melanoma cell line along the proton spread-out Bragg peak. Int J Radiat Biol 2010; 86: 742-51.

12. Absorbed dose determination in external beam radiotherapy: an international code of practice for dosimetry based on standards of absorbed dose to water. IAEA Technical Report Series N 2000; 398: 135-50.

13. Cirrone GAP, Cuttone G, Lojacono PA, et al. A $62 \mathrm{MeV}$ proton beam for the treatment of ocular melanoma at Laboratori Nazionali del Sud-INFN. IEEE Trans Nuc Sci 2004; 51: 860-5.

14. Jonathan EC, Bernhard EJ, McKenna WG. How does radiation kill cells? Curr Opin Chem Biol 1999; 3: 77-83.

15. Valiathan C, McFaline JL, Samson LD. A rapid survival assay to measure drug-induced cytotoxicity and cell cycle effects. DNA Repair (Amst) 2012; 11: 92-8.

16. Berridge MV, Tan AS, McCoy KD, Wang R. The biochemical and cellular basis of cell proliferation assays that use tetrazolium salts. Biochemica 1996; 4: 15-20.

17. Keepers YP, Pizao PE, Peters GJ, van Ark-Otte J, Winograd B, Pinedo HM. Comparison of the sulforhodamine B protein and tetrazolium (MTT) assays for in vitro chemosensitivity testing. Eur J Cancer 1991; 27: 897-900.

18. Banasiak D, Barnetson AR, Odell RA, Mameghan H, Russell PJ. Comparison between the clonogenic, MTT, and SRB assays for determining radiosensitivity in a panel of human bladder cancer cell lines and a ureteral cell line. Radiat Oncol Investig 1999; 7: 77-85.

19. Kim CS, Kim JM, Nam SY, et al. Low-dose of ionizing radiation enhances cell proliferation via transient ERK1/2 and p38 activation in normal human lung fibroblasts. J Radiat Res (Tokyo) 2007; 48: 407-15.

20. Pauwels B, Korst AE, de Pooter CM, et al. Comparison of the sulforhodamine B assay and the clonogenic assay for in vitro chemoradiation studies. Cancer Chemother Pharmacol 2003; 51: 221-6.

21. Ristić-Fira AM, Petrović IM, Korićanac LB, Valastro LM, Privitera G, Cuttone G. Assessment of the inhibitory effects of different radiation qualities or chemotherapeutic agents on a human melanoma cell line. Phys Med 2008; 24: 187-95.

22. Marshall ES, Matthews JH, Shaw JH, et al. Radiosensitivity of new and established human melanoma cell lines: comparison of $(3 \mathrm{H})$ thymidine incorporation and soft agar clonogenic assays. Eur J Cancer 1994; 30A: 1370-6.

23. Griffon G, Merlin JL, Marchal C. Comparison of sulforhodamine B, tetrazolium and clonogenic assays for in vitro radiosensitivity testing in human ovarian cell lines. Anticancer Drugs 1995; 6: 115-23.

24. Daşu A, Denekamp J. Inducible repair and intrinsic radiosensitivity: a complex but predictable relationship? Radiat Res 2000; 153: 279-88.

25. Kraft G, Kramer M. Linear energy transfer and track structure. Adv Radiat Biol 1993; 17: 1-52.

26. Bulat T, Keta O, Korićanac L, Todorović D, Petrović I, Ristić-Fira A. Kinetics of DSB induction and changes in cell cycle regulation in melanoma cells after ionizing radia- 
tion, Proceedings of the $11^{\text {th }}$ International Conference on Fundamental and Applied Aspects of Physical Chemistry; 2012 Sept 24-28; Belgrade, Serbia, 379-81. Society of Physical Chemists of Serbia 2012.

27. Fragkos M, Jurvansuu J, Beard P. H2AX is required for cell cycle arrest via the p53/p21 pathway. Mol Cell Biol 2009; 29: 2828-40.

28. Korićanac L, Žakula J, Cirrone P, et al. Variation of apoptotic pathway regulators by fotemustine and protons in a human melanoma cell line. Adv Sci Lett 2012; 5: 552-9.

29. Arafat WO, Gómez-Navarro J, Xiang J, et al. An adenovirus encoding proapoptotic Bax induces apoptosis and enhances the radiation effect in human ovarian cancer. Mol Ther 2000; 1: 545-54

30. Di Pietro C, Piro S, Tabbì G, et al. Cellular and molecular effects of protons: apoptosis induction and potential implications for cancer therapy. Apoptosis 2006; 11: 57-66.

31. Mielczarek-Palacz A, Kondera-Anasz Z, Sikora J. Higher serum levels of tumour necrosis factor and its soluble receptors are associated with ovarian tumours. Arch Med Sci 2012; 8: 848-53. 